\title{
Integral Sliding mode Control of Height Loop of a Kind of Airship Vehicle
}

\author{
Yuqiang $\mathrm{Jin}^{1}$, Huali Wu and Junwei Lei ${ }^{2}$ \\ ${ }^{1}$ Department of Training, Naval aeronautical and astronautical University \\ Yanti, 264001 \\ ${ }^{2}$ Department of Control engineering, Naval aeronautical and astronautical University \\ Yanti, 264001 \\ ajinyuqiang1024@126.com
}

Keywords: airship; pitch channel; adaptive; sliding mode; stability

Abstract. A kind of integral sliding mode surface method is firstly used in the height loop of airship system based on the assumption that the pitch angle control loop is stable. The expected pitch angle signal is caused by the sliding mode surface which is produced by height error .Compared with traditional PID height loop control strategy, this integral sliding mode method has a very quick output response and simulation result shows that the proposed method is also very accurate in the task of tracking of desired height.

\section{Introduction}

In recent years, due to its potential application value in military, the airship has attracted the attention of researchers from many countries ${ }^{[1-5]}$. The height control loop of the airship is based on the complete design of the inner loop , which is also called attitude angle tracking loop. At present, PID control is widely used in the transformation from height command signal to attitude angle, but the main problem of this method is that the parameters need to be set in advance. It is difficult to achieve a set of parameters for all the desired height ${ }^{[6-10]}$. At the same time, it is difficult to guarantee the rapidness in height control. Based on the above reasons, this paper puts forward a kind of integral sliding mode control method, which can effectively increase the speed and accuracy of the height control due to the introduction of the integral term.

\section{Model Description}

Based on the previous work, the pitch channel model of airship can be described as follows:

$$
M \dot{x}=f(x)+g(x) u
$$

And $x=\left[\begin{array}{llllll}u & w & q & \theta & x & z\end{array}\right], M$ satisfies

$$
M^{-1}=\left[\begin{array}{llllll}
a_{11} & & a_{13} & & & \\
& a_{22} & & & & \\
a_{31} & & a_{33} & & & \\
& & & 1 & & \\
& & & & 1 & \\
& & & & & 1
\end{array}\right]
$$


The definition of $a_{i j}$ see the definition of $M$ in previous work.

Choose the expect value of all states $u, w, q, \theta, x, z$ are $u^{d}, w^{d}, q^{d}, \theta^{d}, x^{d}, z^{d}$, Define the error variable $e=x-x^{d}, \dot{e}=\dot{x}$, then it hold

$$
M \dot{e}=f(x)+g(x) u
$$

Use the inverse matrix of $M$

$$
\dot{e}=M^{-1} f(x)+M^{-1} g(x) u
$$

To make it convenient for reading, some functions can be written as follows

$$
f(x)=\left[\begin{array}{c}
f_{1} \\
f_{2} \\
f_{3} \\
f_{4} \\
f_{5} \\
f_{6}
\end{array}\right] u=\left[\begin{array}{ll}
u_{1} & u_{2}
\end{array}\right]^{T}
$$

where

$$
\left[\begin{array}{c}
f_{1} \\
f_{2} \\
f_{3} \\
f_{4} \\
f_{5} \\
f_{6}
\end{array}\right]=\left[\begin{array}{c}
-\left(m+m_{33}\right) w q+Q\left[C_{X 1} \cos ^{2} \alpha+C_{X 2} \sin (2 \alpha) \sin (\alpha / 2)\right. \\
\left(m+m_{11}\right) q u+m a_{z} q^{2}+Q\left[C_{z 1} \cos (\alpha / 2) \sin (2 \alpha)+C_{z 2} \sin (2 \alpha)+C_{z 3} \sin (\alpha) \sin (\mid \alpha)\right] \\
-m a_{z} w q(-r v)+Q\left[C_{M 1} \cos (\alpha / 2) \sin (2 \alpha)+C_{M 2} \sin (2 \alpha)+C_{M 3} \sin (\alpha) \sin (|\alpha|)\right]-a_{z} \sin \theta W \\
q \\
u \cos \theta+w \sin \theta \\
-u \sin \theta+w \cos \theta
\end{array}\right]
$$

Define

$$
M^{-1} f(x)=\left[\begin{array}{c}
f_{a 1} \\
f_{a 2} \\
f_{a 3} \\
f_{a 4} \\
f_{a 5} \\
f_{a 6}
\end{array}\right]=\left[\begin{array}{c}
a_{11} f_{1}+a_{13} f_{3} \\
a_{22} f_{2} \\
a_{31} f_{1}+a_{33} f_{3} \\
f_{4} \\
f_{5} \\
f_{6}
\end{array}\right]
$$

And

$$
g(x) u=\left[\begin{array}{c}
u_{2} \\
k_{1} u_{1} \\
k_{2} u_{1} \\
0 \\
0 \\
0
\end{array}\right]
$$

Then the system can be written as follows[8-9] 


$$
\left[\begin{array}{c}
\dot{u} \\
\dot{w} \\
\dot{q} \\
\dot{\theta} \\
\dot{x} \\
\dot{z}
\end{array}\right]=\left[\begin{array}{c}
f_{a 1} \\
f_{a 2} \\
f_{a 3} \\
f_{a 4} \\
f_{a 5} \\
f_{a 6}
\end{array}\right]+\left[\begin{array}{c}
a_{11} u_{2}+a_{13} k_{2} u_{1} \\
a_{22} k_{1} u_{1} \\
a_{31} u_{2}+a_{33} k_{2} u_{1} \\
0 \\
0 \\
0
\end{array}\right]
$$

\section{Sliding mode controller design for height loop}

Below design and research is based on the assumption that the pitch angle control system of airship is effective so the goal of height loop is to design the desired or expected value of pitch angle.

Assume the expected height is $Z^{d}$, and define the error variable as

$$
e_{z}=z-z^{d}
$$

Then according to the above model, its derivative can be solved as

$$
\dot{e}_{z}=\dot{z}=-u \sin \theta+w \cos \theta
$$

And choose a integral sliding mode surface as

$$
S_{z}=c_{z} e_{z}+c_{z s} \int e_{z} d t+\dot{e}_{z}
$$

Solve its derivative as

$$
\begin{aligned}
& \dot{S}_{z}=c_{z} \dot{e}_{z}+\ddot{e}_{z}+c_{z s} e_{z}=c_{z} \dot{z}+\ddot{e}_{z}+c_{z s} e_{z} \\
& =-c_{z} u \sin \theta+c_{z} w \cos \theta+(-u \sin \theta+w \cos \theta)^{\prime}+c_{z s} e_{z}
\end{aligned}
$$

where

$$
(-u \sin \theta+w \cos \theta)^{\prime}=-\dot{u} \sin \theta+\dot{w} \cos \theta-(u \cos \theta+w \sin \theta) q
$$

then

$$
\dot{s}_{z}=-c_{z} u \sin \theta+c_{z} w \cos \theta-\dot{u} \sin \theta+\dot{w} \cos \theta-(u \cos \theta+w \sin \theta) q+c_{z s} e_{z}
$$

Design the expected value of pitch angle as

$$
\theta=\left[k_{z 1} s_{z}+k_{z 2} s_{z} /\left(\left|s_{z}\right|+\xi\right)\right]+d_{t} \operatorname{sign}\left(s_{z}\right)
$$

And define

$$
\Delta=c_{z} w \cos \theta-\dot{u} \sin \theta+\dot{w} \cos \theta-(u \cos \theta+w \sin \theta) q+c_{z s} e_{z}
$$

where

$$
\dot{S}_{z}=-C_{z} u \sin \left[k_{z 1} s_{z}+k_{z 2} s_{z} /\left(\left|s_{z}\right|+\xi\right)\right]+\Delta
$$

For the following inequality, there exist positive constant $k_{p 1}$ and $k_{p 2}$ such that 


$$
k_{p 1} \theta^{2} \leq \theta \sin \theta \leq k_{p 2} \theta^{2}
$$

For a small $\theta$, when $\Delta$ compare with $\theta$, it can satisfy $\Delta<d_{r} \theta$, so

$$
\theta \dot{s}_{z}=-c_{z} \theta u \sin [\theta]+\Delta \theta
$$

Then it holds

$$
\left[k_{z 1}+k_{z 2} /\left(\left|s_{z}\right|+\xi\right)\right] s_{z} \dot{s}_{z} \leq-C_{z} u k_{p 1} \theta^{2}+d_{r} \theta^{2}
$$

So it is easy to choose proper control gian such that

$$
S_{z} \dot{S}_{z} \leq 0
$$

And according to Lyapunov stable theorem, the system is stable.

\section{Numerical simulation}

To testify the rightness of the above method, a numerical simulation is done as follows: choose control parameter as $c_{z}=0.06$, set $k_{z 1}=0.1, k_{z 2}=5, \xi=10$, then assume $u=20 \mathrm{~m} / \mathrm{s}, T_{m}=8000 \mathrm{~N}$, initial position $x_{e}=y_{e}=z_{e}=0$. Other quantity of state is 0 , choose $z_{d}=-500$, simulation results are as following figures:

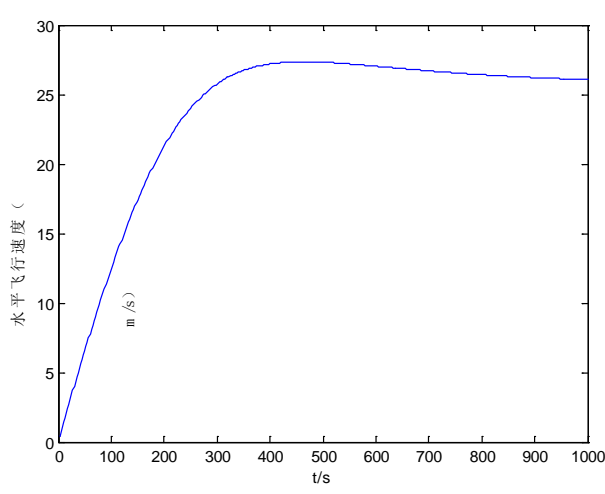

Fig.2 Forward Velocity

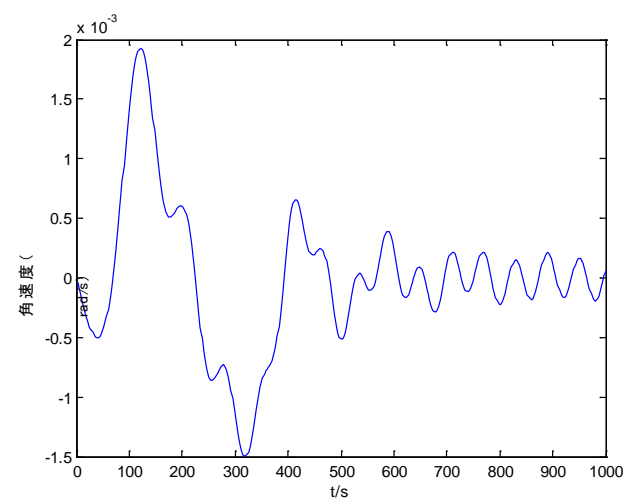

Fig. 4 Angle Velocity

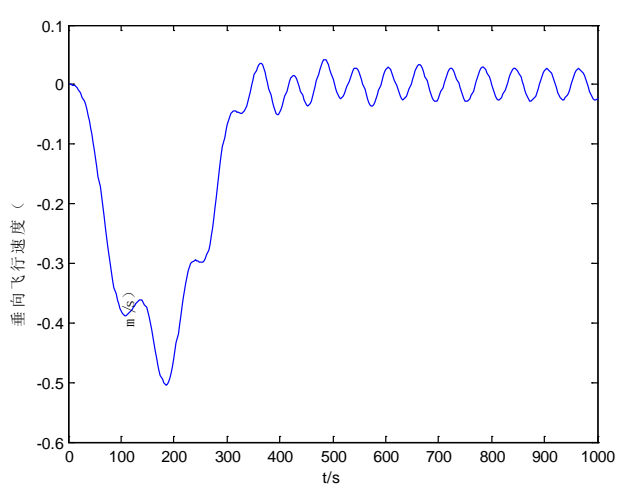

Fig.3 Vertical Velocity

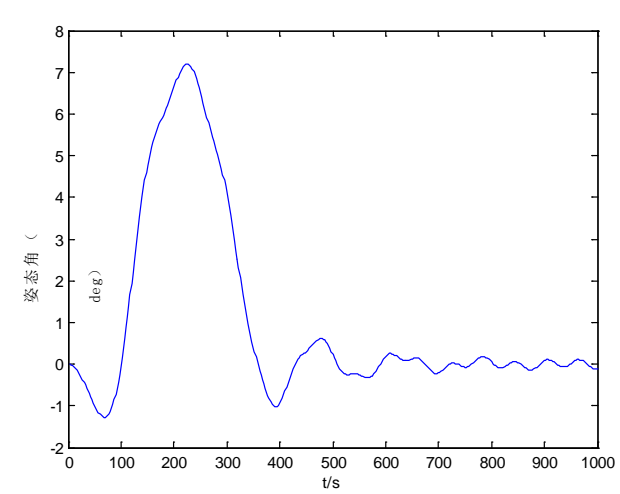

Fig.5 Pitch Angle 


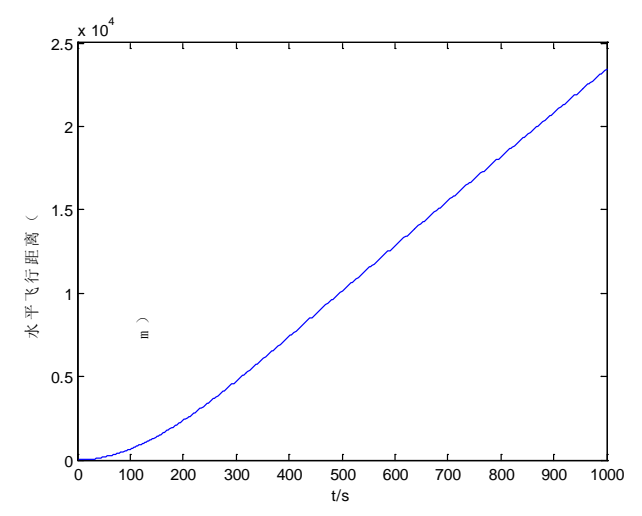

Fig. 6 Flying Distance

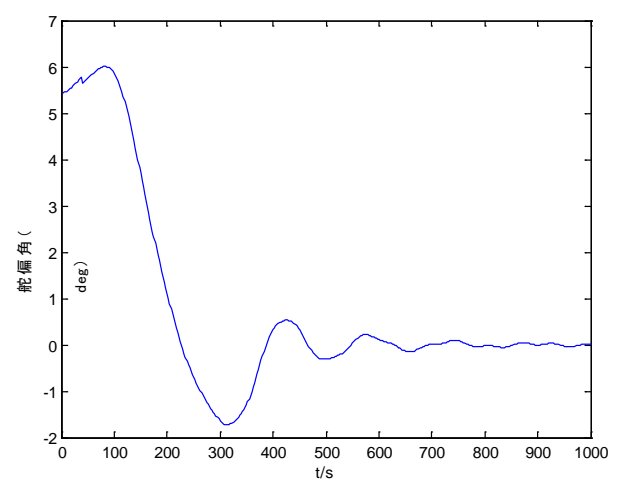

Fig. 8 Pitch Actuator Angle

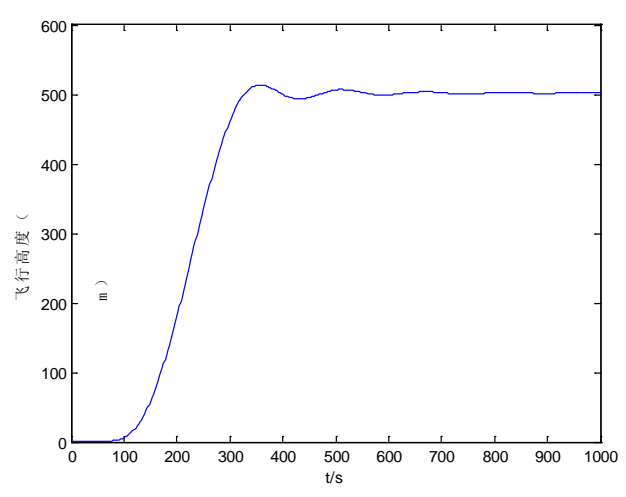

Fig. 7 Height

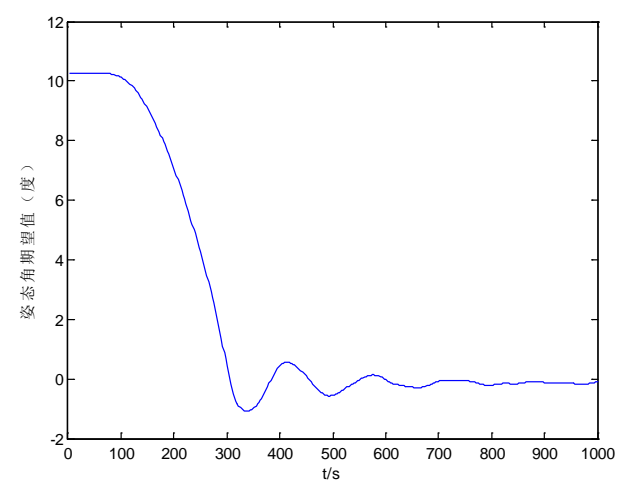

Fig. 9 Expected value of pitch angle

The simulation results show that airship can follow the tracks of expected altitude 500s, and the speed of airship is about $25 \mathrm{~m} / \mathrm{s}$, and the rise time of response of height loop is about 200s, which is very quick for an airship system, so simulation result shows that the height control system is effective and stable.

\section{Conclusion}

A kind of integral sliding mode method is firstly used to generate the command signal of pitch angle which can make the height of airship track the desired value. So this novel method is totally different from traditional height control method such as PID method. And the advantage which is showed by the detailed simulation is that the tracking of desired height can be a very big value and the actuator angle can also avoid saturation problem, also the response is very quick and accurate.

\section{References}

[1] J.S.Uhlman,N.E.Fine,D.C.Kring.Calculation of the Added Mass and Damping Forces on Supercavitating Bodies.The 4th International Symposium on Cavitation,California,2001:7 13

[2] D.Clarke.Calculation of the Added Mass of Elliptical Cylinders in Shallow Water.Ocean Engineering.2001,28(4):61 72

[3] C.J.Atkinson,R.G.Urso.Modeling of Apparent Mass Effects for the Real-Time Simulation of a Hybird Airship.AIAA Modeling and Simulation Technologies Conference and Exhibit,Keystone.2006:21 32

[4] Yokomaku Y.The Stratospheric Platform Airship R\&D Program of Japan. The 2nd Stratospheric Platform Systems Workshop,Tokyo Japan.2000:7 13 
[5] S.P.Jones,J.D.Laurier.Aerodynamic Estimation Techniques for Aerostats and Airships.AIAA Lighter-than-Air Systems Conference,Annapolis,2004: 88 94

[6] M.T.Soylemeza,N.Munrob,H.Bakic.Fast Calculation of Stabilizing PID Controllers.Automatic,2003,39(7):121 126

[7] Etkin B, Theory of the flight of Airplanes in Isotropic Turbulence Review an Extension,AGARD Rept.1961:372

[8] David K. Schmidt,James Stevens,Jason Roney.Dynamic Modeling,Control, and Station-Keeping Guidance of A Large High-Altitude“Near-Space” Airship.AIAA Guidance,Navigation,and Control Conference and Exhibit. 2006-6781

[9] David K. Schmidt.Modeling and Near-Space Station-Keeping Control of a Large High-Altitude Airship.Journal of Control and Dynamics,2007,30(2):540 547

[10] Azinheira, J.R., A.Moutinho, Hover Control of an UAV With Backstepping Design Including Input Saturations[J]. IEEE Transactions on Control System Technology, 2008.16(3):517-526 